\title{
Experiment on Wingtip Vortices using a Half Deltawing at the Tips
}

\author{
Muthu Kumar S
}

\begin{abstract}
The counter rotating wing tip vortices produced by the aircraft continues to be a big concern for the aviation industry and the aircraft manufacturers due to its hazardous effects on the flight safety and aircraft efficiency. The strength of the vortices poses severe problems to the aircraft operations. Manufacturers developed various wingtip devices to alleviate this problem, but still it is not fully understood and solved. In this thesis, the effectiveness of using a half delta wing at the tips is investigated. The flow field over a low aspect ratio NACA 0015 wing fitted with a slender sharp half delta wing with a leading edge sweep angle 700 at a Reynolds number $1.87 \times 105$ is investigated. Particle image velocimetry is used to quantify the vortex structure and force balance measurements are used to calculate the aerodynamic data of the wing. The peak vorticity, peak tangential velocity are decreased due to the addition of half delta wing. The over-all radius of the wingtip vortex increased showing a diffused vortex due to the addition of the half delta wing. The core circulation is decreased leading to a lower strength vortex. Though the tip device increased the drag, it increases the aerodynamic efficiency through the improvement in L/D.
\end{abstract}

Index Terms -Wingtip vortices,wind tunnel testing,PIV,Induced drag,circulation,Delta wing

\section{INTRODUCTION}

When a lifting surface like finite wing or rotor blades generates aerodynamic lift due to the pressure difference between the upper and lower surface, it causes the airflow to go around the tip from pressure side to the suction side. This process causes the circulatory flow pattern named vortex and it is called as the wingtip vortex or tip vortex depending up on the lifting surface which produces it. In case of the finite wing a pair of counter rotating vortices are formed at the tips. These vortices are characterized by high levels of vorticity and the strength of the vortices depends on the lift produced by the aircraft.

Wingtip vortex is the term mainly used for the vortex structure formed at the tips of the aircraft wing, however the same process could be witnessed in the rotor blades of helicopters and propellers. They are visible naturally, when the condensation of water vapour happens in air. In the field of aeronautics, safety has been given a higher priority and the technologies developing are mainly concerned about ensuring safe and efficient flight.

The strength of the vortical structure depends on the lift produced by the aircraft. During take-off and landing, if an aircraft flies in to the wake generated by the preceding aircraft, it would face a serious hazard and particularly dangerous since it is happening near the ground. The aircraft will be imposed with roll, loss of altitude and strong

Revised Manuscript Received on August 19, 2019.

MuthuKumar S, Department of Aeronautical Engineering, Kumaraguru College of Technology, Coimbatore, Tamil Nadu, India. (E-mail: muthukumar.s.aero@kct.ac.in) structural loads. This will be serious when a smaller aircraft follows a bigger one. Bobylev2010 [1] has presented the calculations of the wake characteristics for certain aircrafts and safe distance values during landing with various turbulent atmospheric states.

To avoid this problems of wake encountering, during take-off and landing, the regulatory board defines a minimum distance between the aircrafts depending up on its size. As a result of this wake encountering problem, the operating cost of the airlines and airport increased severely.

The tip vortex which is detached from the trailing edge starts interacting with the wake vorticity and rolls in to a vortex. The forming and roll-up process happens in the near field region. The vortex is neither fully developed nor axisymmetric in the near field. The investigation on formation of the vortices is more important as it is responsible for the far field stronger vortex. Wingtip modification affects the formation and the rolling up process. Grow et al [9] used a five hole pressure probe and a vorticity meter to investigate the wingtip vortices. This work shows that there is an increase in circulation and maximum tangential velocity with the increase in aspect ratio and angle of attack. Orloff [10] used laser Velocimetry to measure the vortex at $\mathrm{x} / \mathrm{c}=2$ with NACA 0015 aerofoil. $\mathrm{He}$ describes the defects in using the point measurement devices asit would affect the vortex structure. It was observed that there is a defect and an excess of axial velocity at the vortex centre at an angle of attack 8 and 12 degrees. Francis et al [11] worked on the vortex development in the near field region. He used a thin laser sheet and a dye to visualize the vortices. He mentioned about the presence and persistence of secondary vortices at the square tip along with the counter rotating vortices. Shekarriz [12] used the particle image Velocimetry (PIV) to investigate the vortex system and observed that circulation remains constant from $\mathrm{x} / \mathrm{c}=1$ to $\mathrm{x} / \mathrm{c}$ $=6.7$ downstream from the trailing edge. He also mentions that the near field is completely dominated with secondary vortices.Shekarriz [12] used the particle image Velocimetry (PIV) to investigate the vortex system and observed that circulation remains constant from $\mathrm{x} / \mathrm{c}=1$ to $\mathrm{x} / \mathrm{c}=6.7$ downstream from the trailing edge. He also mentions that the near field is completely dominated with secondary vortices. Davenport et al [5] had done a significant work in detecting the structure of the tip vortices at the near field. He performed experiments on NACA 0012 half wing using hot wire probe and addressed the wandering of vortices. A theory was formulated to develop correction for this wandering 
phenomenon. He observed that the flow outside the vortices are dominated by the wing wake and the core is laminar. The velocity fluctuations inside the core are due to the turbulence presented in the surrounding spiral wake. Birch et al [13] used PIV, 7 hole pressure probe and force balance for measuring the trailing vortex on a wing by varying the tip geometry. It was observed that the core radius, peak vorticity, peak tangential velocity increases with angle of attack. Due to increased reliability, simplicity and accuracy, PIV technique has gained the reputation of a powerful and time effective flow diagnostic method for the quantitative flow field study of complex fluid flows. Corsiglia et al [14] investigated NACA 0015 wing at $\operatorname{Re}=9 \times 10^{5}$ at $\alpha=12^{0}$ and observed that the normalized tangential velocity decreased from 0.42 to 0.12 as they moved from $\mathrm{x} / \mathrm{c}=1$ to $\mathrm{a}$ downstream location of $\mathrm{x} / \mathrm{c}=5$. He suggested that this decrease is due to the interaction of the vortex with the aircraft wake.

\section{EXPERIMENTAL SETUP}

\section{A. Wind tunnel and model}

The experiment was conducted in the low speed low turbulence wind tunnel. This is an open circuit continuous ow wind tunnel in the low speed aerodynamics lab of IIT Kanpur. It is a 3D sectioned wind tunnel.

Detailed specifications are:

Test section: $0.6 \mathrm{~m} \times 0.6 \mathrm{~m} \times 3 \mathrm{~m}$

Contraction ratio: 16

No of mesh screens: 3

Maximum free stream velocity: $40 \mathrm{~m} / \mathrm{s}$

Fan type: 4 bladed propeller fan of $1.5 \mathrm{~m}$ diameter

Power supply: single 25 hp DC motor

The wing model with NACA 0015 aerofoil designed particularly for force measurement with explicit force balance mounting provisions. The model was fabricated with the help of rapid prototyping technique using ABS plastic from $4 \mathrm{i}$ lab facility of IIT Kanpur. Model was made in 9 sectional pieces and joined separately on a common shaft along the span of aerofoil model. Separate adapters were made of stainless steel from the aerospace workshop for housing the 5 component force balance. The adapter fixes the model to the tunnel wall. The half delta wing was made of Perspex. The model specifications is given in figure 2

\section{B. Instrument and measurement chain}

A 5 component internal strain gauge balance is used in the experiment to measure the forces. The arrangement of model with the balance on the tunnel wall is shown in figure 3. A clearance of $1 \mathrm{~mm}$ between the model and the wall is provided, so as to allow the model to entirely rest on the end of the balance.

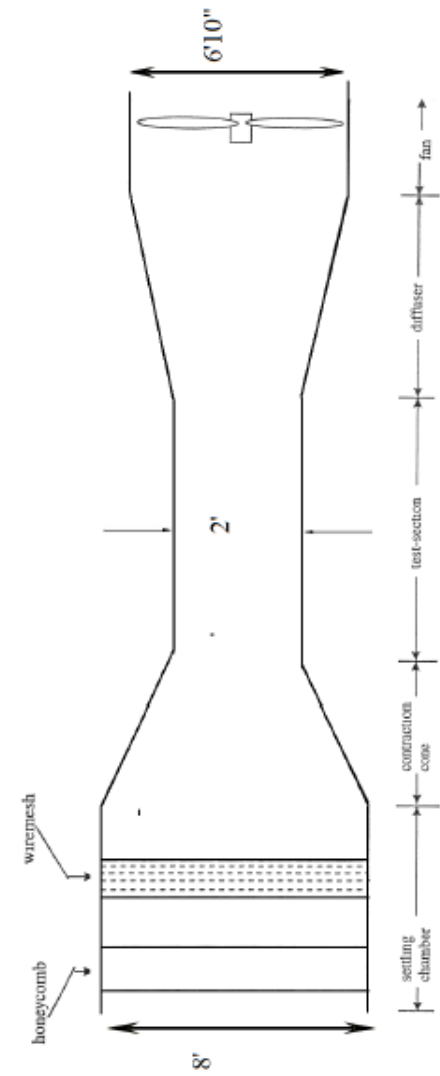

Fig. 1 Wind tunnel facility

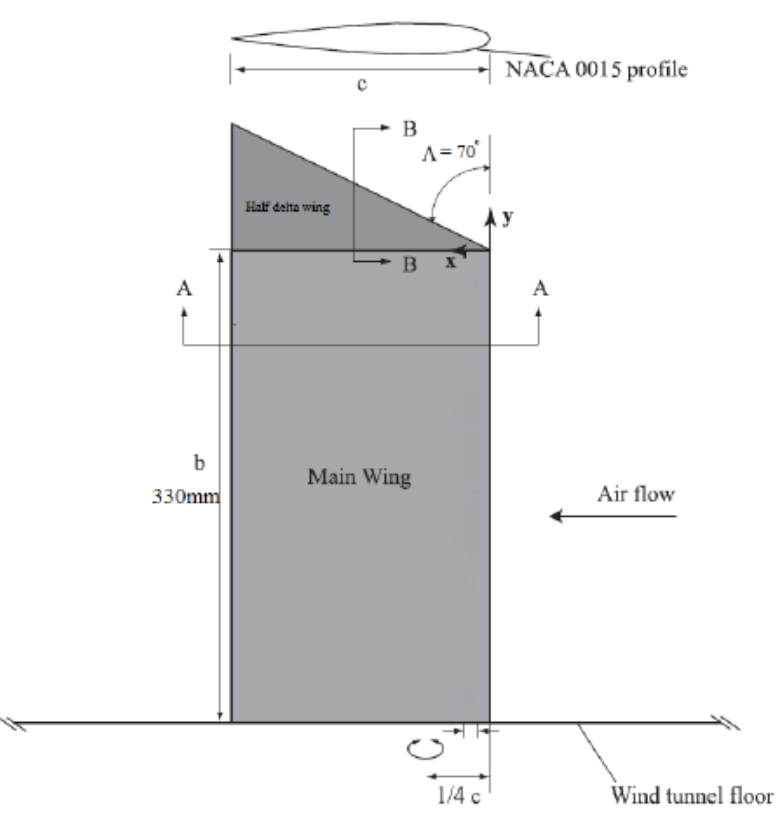

Fig. 2 Wind tunnel model

Multi-function data acquisition board (NI-PXI-6251 Mseries) from the National Instruments Inc. USA was used to acquire data from the 5 component force balance. It acts as the interface between the computer and the strain gauge balance. 
It has 18 bit $\mathrm{ADC}$ to digitize the data. It has a high resolution that could resolve smaller forces. It can acquire data from 32 channels in single ended mode and 16 channels in differential mode at a maximum sampling rate of 500 $\mathrm{kS} / \mathrm{s}$. It has analog and digital triggering option to start or stop acquisition. Data acquisition board are routed through signal conditioning board NI-SCXI-1520.

Signal conditioner is the key element which provides distinct enhancement to both performance and accuracy of data. Amplification, attenuation, isolation, filtering, excitation, bridge completion are some of the processes done by signal conditioner. In our case the signals from the strain gauge were in millivolts $(\mathrm{mV})$ and an excitation of 4.25 volts were provided by the signal conditioner. NISCXI-1520 housed in the chassis NI-SCXI-1000 was used. Filter setting was set to $10 \mathrm{~Hz}$.

PIV has high spatial resolution, but probes has high temporal resolution. Temporal resolution is limited by the laser_ring capacity. TSI PIV system was used during the experiment. It consists of $200 \mathrm{MJ} \mathrm{Nd}-\mathrm{YAG}$ laser and synchronizer. Flow was seeded with Antari 23000 ii series fog generator using Antari FLG fog fuel. Image processing was done using the Insight $3 \mathrm{G}$ software. Tecplot software was used in visualize the velocity and vorticity fields. The schematic of the PIV system is shown in figure 4

The imaging system for the PIV system consists of a power view 4 MP CCD camera. Camera was triggered by the synchronizer and image of the particles illuminated were taken. These light energy were converted to electrical energy by CCD camera and send to the frame grabber which resides in the computer. Camera lens of $50 \mathrm{~mm}$ was used in the experiment.

Usually fog of 1 to 10 micron is used in case of air medium. In the present experiment we have used the Z30011 fog series as the seeding particles. It gives fog around 3-5 microns. Fog generator was kept outside the inlet section of the open circuit wind tunnel. The smoke generator is operated intermittently and a small table fan was used to spread the smoke to get uniformity over the test section.

\section{EXPERIMENTAL PROCEDURE}

Two measurement techniques were used to study the effects of wingtip vortices. Particle image Velocimetry (PIV) to measure the vortex structure and complete characterization of the vortices. A 5 component strain gauge balance is used to measure the aerodynamic forces on the model by changing the angle of attack and keeping the Reynolds number constant.

Force data is acquired using a 5-component internal strain gauge balance. The data acquired at a sampling rate of 200 . The filter setting was set as $10 \mathrm{~Hz}$ to eradicate the antialiasing problem. An excitation voltage of $4.25 \mathrm{~V}$ is given for the bridge configuration from the signal conditioner. The data were acquired using data acquisition board (NI-PXT 6251). The signals from the balance are routed through the signal conditioner (NI-SCXI-1520). It is where the signal gets amplified, filtered and sent to through DAQ board. Data from the active channels are acquired and stored for further analysis. Data without the free stream velocity was acquired for all the angle of attacks and subtracted from the wind data acquired for the further force calculations for more accuracy. The signals acquired from balance were in millivolts and it was normalized with the excitation voltage of 4.25 provided by the signal conditioner resulting in signals of unit $\mathrm{mV} / \mathrm{V}$. These five channel signals are acquired for 5 seconds with a scan rate of 200 and averaged. After normalization the signals are to be converted in to normal, side forces and rolling moment. The inverse matrix obtained from the calibration is multiplied with the acquired signal.

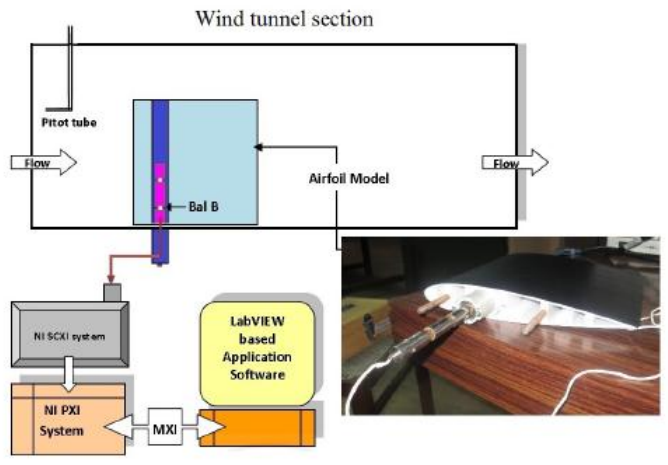

Fig. 3 Model mounting with the balance

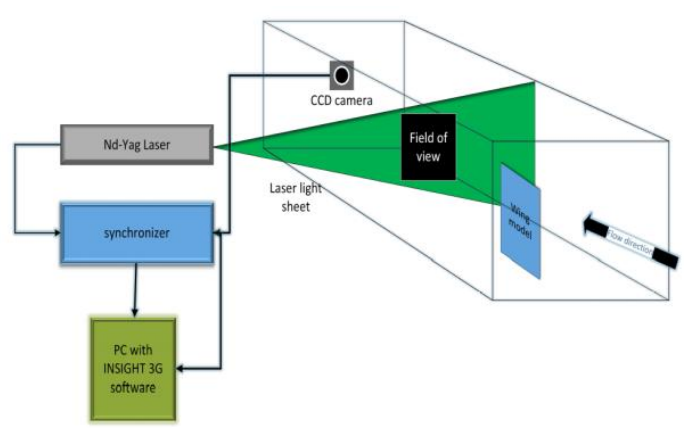

Fig. 4 PIV schematic

The equation for conversion is

$\mathrm{F}_{\mathrm{ij}}=\mathrm{C}_{\mathrm{ij}} \mathrm{E}_{\mathrm{ij}}$

$F_{i j}=N_{1} ; N_{2} ; S_{1} ; S_{2} ; R_{m}$

$\mathrm{E}_{\mathrm{ij}}=\mathrm{E}_{\mathrm{N} 1} ; \mathrm{E}_{\mathrm{N} 2} ; \mathrm{E}_{\mathrm{S} 1} ; \mathrm{E}_{\mathrm{S} 2} ; \mathrm{E}_{\mathrm{Rm}}$

Fij ;Eij are the forces and signals respectively

All the forces are calculated about the balance centre and the total normal andside force are calculated as follows

$$
\begin{aligned}
& \mathrm{N}=\mathrm{N} 1+\mathrm{N} 2 \\
& \mathrm{~S}=\mathrm{S} 1+\mathrm{S} 2 \\
& \mathrm{M}=\mathrm{R}_{\mathrm{m}}
\end{aligned}
$$

These loads are transferred to the wind axis system as Normal force $=-\mathrm{N}$

Side force $=\mathrm{S}$

Normal force acting perpendicular to the chord line and Side force acting along the chord towards the trailing edge. The lift and drag forces are perpendicular and parallel to the free stream hence these are calculated from the normal and side forces as follows 


$$
\begin{aligned}
& \mathrm{L}=\mathrm{N} \cos \alpha-\mathrm{S} \sin \alpha \\
& \mathrm{D}=\mathrm{N} \sin \alpha+\mathrm{S} \cos \alpha
\end{aligned}
$$

Area $S=273 \mathrm{~mm} \_303 \mathrm{~mm}$ (in case of base wing)

Area $S=$ Area of base wing + Area of half delta wing (in case of base wing equipped with half delta wing)

Each acquisition consists of two images with the time interval $\Delta \mathrm{t}$ between the laser pulses. Each image is divided in to interrogation windows of size $32 \times 32$ pixels and each image is cross correlated with the corresponding region. The highest peak in the cross correlation image is most likely the displacement vector and it is the one which is used to calculate the velocity vector of the interrogation window. The field of camera is $270 \mathrm{~mm} \times 270 \mathrm{~mm}$. the total pixels in the camera is 2048 and it is distributed among this field of view. Instantaneous velocity samples are acquired and a averaging of 150 such samples are done for a better data.

\section{RESULTS AND DISCUSSIONS}

\section{A. Rankine vortex}

Rankine vortex is theoretical model of vortex in viscous fluid. A swirling flow in a viscous fluid can be characterized by a forced vortex in its central core surrounded by a free vortex. On the other hand, in an Inviscid fluid the flow consists entirely of the free vortex with an infinite velocity at the center. From the Rankine vortex model it is clear that the central forced vortex has the profile, which shows an increase in swirl velocity with the radius of the vortex. Vortex core is defined as the radius at which the maximum swirl velocity is obtained. After the point of maximum, the swirl velocity starts decreasing with the radius. In this region the viscous effects are dominant. The vorticity produced at the wings boundary layer goes in to the vortex core.

\section{B. Comparison of Rankine vortex with the experimental data}

As per the theoretical Rankine vortex model there will be a sharp peak occurring in the tangential velocity profile is shown in figure 5 , but in the experimental data shown in figure 7 , shows the absence of sharp peak, but only a smoothvariation is detected. Theoretically the vorticity produced on the solid body goes into the core of the vortex. The free vortex rotating will be irrotational, but the experimental results show traces of vorticity outside the core also. Though the finite amount of vorticity is seen outside of the core, about $70 \%$ of the vorticity is inside the core only. The location ofvorticity peak is taken as the vortex center.

The vortices are captured at a downstream location of $\mathrm{x} / \mathrm{c}$ $=2$. This region is away from the near field hence it is free from the secondary vortices which is dominant in the near field. At this location the roll-up process is almost complete and an axisymmetric vortex is formed. But in our case the vortex has reached a near axisymmetric structure which is visible through the swirl velocity profile. The vortices were capture by changing the angle of attack and keeping the Reynolds number constant.

Throughout these results, the normalization process is done as follows:

1. The vorticity $\xi$ is normalized with wing chord $\mathrm{C}$ and free stream velocity $\mathrm{u}_{\infty}$
2. The swirl velocity $\mathrm{V}_{\theta}$ is normalized with the free stream velocity $\mathrm{u}_{\infty}$

\section{Vorticity distribution and comparison with the base wing}

The distribution of vorticity is different in the case of the half delta wing. The isovorticity contours for different angle of attacks is given in figure 6 and figure 8 . It can be even visually seen that the vortex is more am more diffused. The core region has a lower vorticity than the base wing. The vortex axial velocities are either jet like or wake like [8]. After leading edge vortex breakdown the delta wingtip vortices exhibit wake like behavior which would have led to the diffused vortex.

The base wing vortices has linear increase in vorticity peak, but in the case of half delta wing, the peak vorticity increases from $\alpha=2^{0}$ to $\alpha=10^{0}$. It reaches its maximum value at $\alpha=10^{\circ}$ and starts decreasing slowly after that point. The maximum vorticity reaches $\xi \mathrm{c} / \mathrm{u}_{\infty}=33.42$ which is $50 \%$ of the peak vorticity value of base wing. This may be due to the vortex breakdown occurs at the half delta wing which lead to the more diffused vortex with less peak vorticity. The vorticity distribution for the entire angle of attacks is shown in the figure 8 . It is evident form the figure 10,that the peak vorticity value of half delta equipped wing is lower for all the angle of attacks compared to the base wing.

\section{Swirl velocity distribution and comparison with the base Wing}

The swirl velocity distribution across the vortex centre is given in figure7 \& 9 . The maximum tangential velocity is obtained at an angle of attack $\alpha=15^{\circ}$. It ranges from -0.389 to 0.459 . The axisymmetric structure is same as the base awing and the peak tangential velocity is half of the value of base wing. The swirl velocity is an important parameter in the vortex hazard encountering. If an aircraft with a lower aileron power gets in to the vortex may go out of control due to the high swirl velocity present in it the vortex. In that case this half delta wing would be a useful one as it reduces the peak tangential velocity in to half the value of base wing. As the angle of attack increases the peak tangential velocity also increases linearly. The comparison between base and half delta equipped wing is shown in _gure 6.26. The result is also supplemented with the experimental works of birch et al [13] and it has good match with our experimental data.

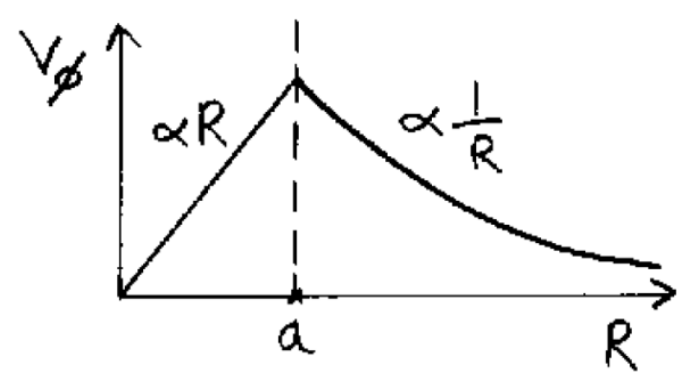

Fig. 5 Model mounting with the balance 


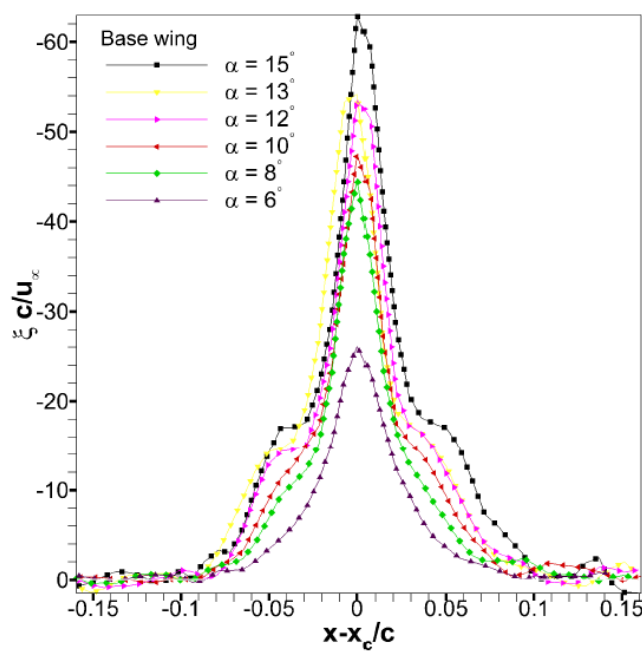

Fig. 6 Vorticity Distribution for different angle of attack at $\operatorname{Re}=1.87 \times 10^{5}$

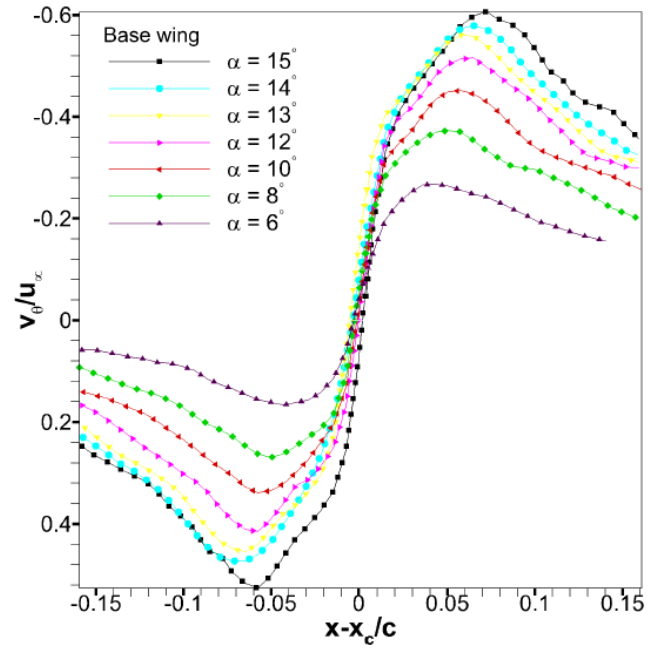

Fig. 7 Tangential velocity Distribution for different angle of attack atRe $=1.87 \times 10^{5}$

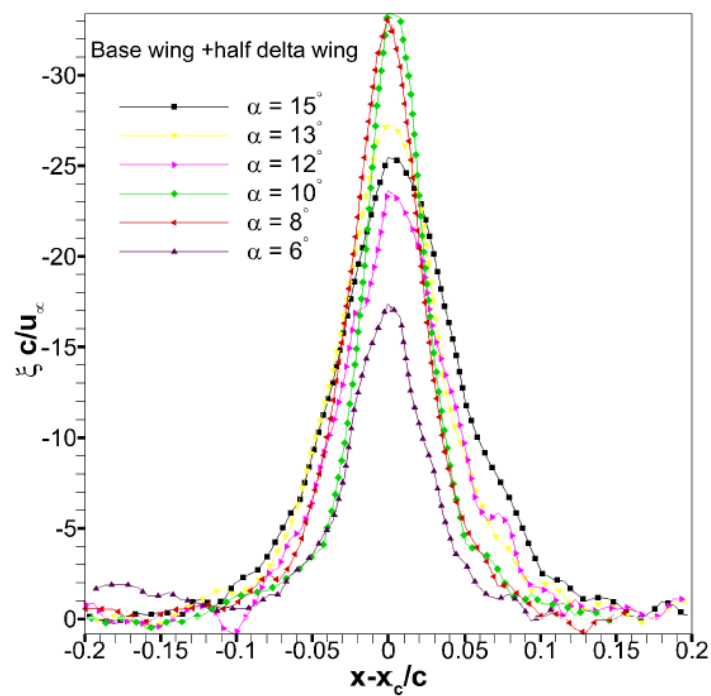

Fig. 8 Distribution of vorticityacoross the vortex $R e=$ $1.87 \times 10^{5}$

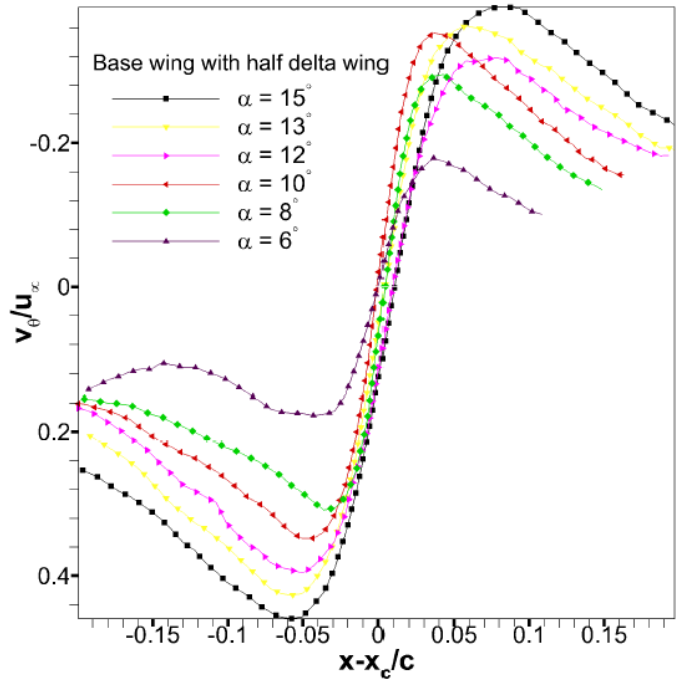

Fig. 9 Distribution of Tangential velocity across the vortex at $\operatorname{Re}=1.87 \times 10^{5}$

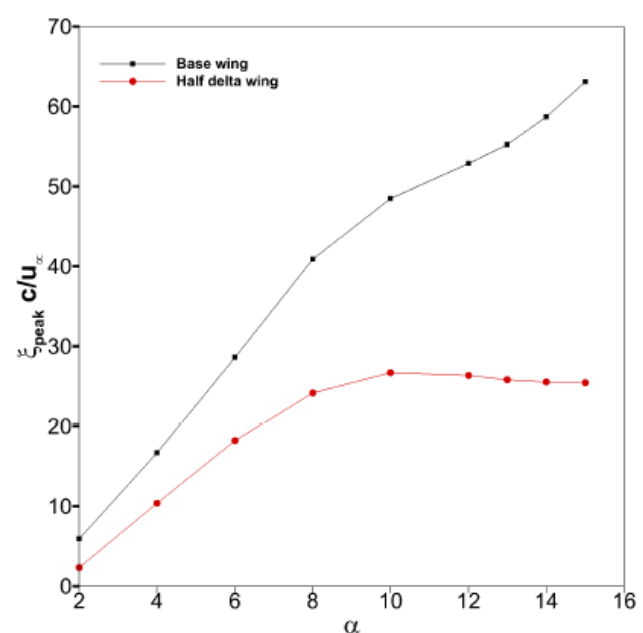

Fig. 10 Variation of peak vorticity with angle of attack $\operatorname{Re}=.87 \times 10^{5}$

\section{CONCLUSION}

Experiment has been conducted on a 3D wing with NACA 0015 aerofoil. Effectiveness of adding a half delta wing to the tips is investigated and got the following conclusions. The peak vorticity value got decreased $50 \%$ when the half delta wing is attached to the tips, which shows the diffused vortex. The half delta equipped wing has a smaller core radius but a higher overall vortex radius compared to the base wing. It shows the diffusion of vorticity from the core to the outer part of the vortex. The core circulation created by the half delta equipped wing is less than the base wingtip vortices, proving that the strength of the vortices is reduced when the half delta wing is attached. The half delta wing has diffused the vortex to bring down its strength. Further the work could be extended by measuring the vortex characteristics at various other locations. 


\section{REFERENCES}

1. Bobylev, A. V., V. V. Vyshinsky, G. G. Soudakov, and V. A. Yaroshevsky (2010) \Aircraft Vortex Wake and Flight Safety Problems," Journal of Aircraft, 47(2), pp. 663-674.

2. Anderson, J. D. (2001) Fundamentals of aerodynamics, vol. 2, McGraw-Hill New York.

3. Francis, M. S. and D. A. Kennedy (1979) "Formation of a trailing vortex," Journal of Aircraft, 16(3), pp. 148-154.

4. Chow, J. S., G. G. Zilliac, and P. Bradshaw (1997) "Mean and turbulence measurements in the near field of a wingtip vortex," AIAA journal, 35(10), pp. 1561-1567.

5. Devenport, W. J., M. C. Rife, S. I. Liapis, and G. J. Follin (1996) "The structure and development of a wing-tip vortex," Journal of Fluid Mechanics, 312(67), p. 106.

6. Agalya, A., B. Nagaraj, and K. Rajasekaran. "Concentration control of continuous stirred tank reactor using particle swarm optimization algorithm." Trans EngSci 1, no. 4 (2013): 57-63.

7. Gerz, T., F. Holz $\square$ apfel, and D. Darracq (2002) "Commercial aircraft wake vortices," Progress in Aerospace Sciences, 38(3), pp. 181-208.

8. Lee, T. and J. Pereira (2008) \Tip vortex control via a tab deecting athigher harmonic frequencies," AIAA journal, 46(6), pp. 1342-1350.

9. GROW, T. L. (1969) $\backslash E_{-}$ect of a wing on its tip vortex." Journal of Aircraft, 6(1), pp. 37-41.

10. Orloff, K. L. (1974) "Trailing vortex wind-tunnel diagnostics with a laser velocimeter," Journal of Aircraft, 11(8), pp. 477-482.

11. Francis, M. S. and D. A. Kennedy (1979) "Formation of a trailing vortex," Journal of Aircraft, 16(3), pp. 148-154.

12. Shekarriz, A., T. Fu, J. Katz, and T. Huang (1993) Near-_eld behaviorof a tip vortex," AIAA journal, 31(1), pp. 112-118.

13. Birch, D., T. Lee, F. Mokhtarian, and F. Kafyeke (2003) "Rollup and near-_eld behavior of a tip vortex," Journal of aircraft, 40(3), pp. 603 607.

14. Corsiglia, V., R. Schwind, and N. Chigier (1973) "Rapid Scanning, Three-Dimensional Hot-Wire AnemometerSurveys of Wing-Tip Vortices," Journal of Aircraft, 10(12), pp. 752-757.

15. Hoffmann, E. R. and P. N. Joubert (1963) "Turbulent line vortices," Journal of Fluid Mechanics, 16, pp. 395-411.

16. Patterson, J. C. (1975) "Vortex Attenuation Obtained in the Langley Vortex Research Facility," Journal of Aircraft, 12(9), pp. 745-749.

17. Corsiglia, V. R., R. A. Jacobsen, and N. Chigier (1971) "An experimental investigation of trailing vortices behind a wing with a vortex dissipator," in Aircraft Wake Turbulence and Its Detection, Springer, pp. 229-242.

18. Myose, R., M. Papadakis, and I. Heron (1998) "Gurney flap experiments on airfoils, wings, and reection plane model," Journal of Aircraft, 35(2), pp. 206-211.

19. Lee, T. and D. M. Birch (2006) "Oscillating-wing tip vortex with passiveshort-span trailing-edge aps," Journal of aircraft, 43(3), pp. 723-731.

20. Duraisamy, K. and J. D. Baeder (2005) "Numerical simulation of the effects of spanwise blowing on wing-tip vortex formation and evolution," AIAA Paper, 4726, p. 2005. 